Draft VERSion July 8, 2018

Preprint typeset using LATEX style AASTeX6 v. 1.0

\title{
A SEARCH FOR PHOTOMETRIC VARIABILITY IN THE YOUNG T3.5 PLANETARY-MASS COMPANION GU PSC b
}

\author{
Marie-Eve Naud $^{1 *}$, Étienne Artigau ${ }^{1}$, Jason F. Rowe ${ }^{1}$, René Doyon $^{1}$, Lison Malo ${ }^{2,1}$, Lö̈c Albert ${ }^{1}$, Jonathan $^{2}$ \\ GAGnÉ $^{3,4}$, AND SANDiE BouChard ${ }^{1}$ \\ ${ }^{1}$ Institut de recherche sur les exoplanètes, Département de physique, Université de Montréal, Montréal, QC H3C 3J7, Canada. \\ 2 Canada-France-Hawaii Telescope, 65-1238 Mamalahoa Hwy, Kamuela, HI 96743, USA. \\ ${ }^{3}$ Department of Terrestrial Magnetism, Carnegie Institution for Science, 5241 Broad Branch Road NW, Washington, DC 20015, USA. \\ ${ }^{4}$ NASA Sagan Fellow.
}

\begin{abstract}
We present a photometric $J$-band variability study of GU Psc b, a T3.5 co-moving planetary-mass companion (9-13 $\left.M_{\mathrm{Jup}}\right)$ to a young $(\sim 150 \mathrm{Myr}) \mathrm{M} 3$ member of the AB Doradus Moving Group. The large separation between GU Psc b and its host star $\left(42^{\prime \prime}\right)$ provides a rare opportunity to study the photometric variability of a planetary-mass companion. The study presented here is based on observations obtained from 2013 to 2014 over three nights with durations of $5-6 \mathrm{hr}$ each with the WIRCam imager at Canada-France-Hawaii Telescope. Photometric variability with a peak-to-peak amplitude of $4 \pm 1 \%$ at a timescale of $\sim 6 \mathrm{hr}$ was marginally detected on 2014 October 11 . No highsignificance variability was detected on 2013 December 22 and 2014 October 10. The amplitude and timescale of the variability seen here, as well as its evolving nature, is comparable to what was observed for a variety of field $\mathrm{T}$ dwarfs and suggests that mechanisms invoked to explain brown dwarf variability may be applicable to low-gravity objects such as GU Psc b. Rotation-induced photometric variability due to the formation and dissipation of atmospheric features such as clouds is a plausible hypothesis for the tentative variation detected here. Additional photometric measurements, particularly on longer timescales, will be required to confirm and characterize the variability of GU Psc b, determine its periodicity and to potentially measure its rotation period.
\end{abstract}

Keywords: planets and satellites: atmospheres - planets and satellites: gaseous planets - stars: individual (GU Psc) - techniques: photometric

\section{INTRODUCTION}

The study of photometric variability is a unique and powerful technique to explore the nature and dynamics of exoplanet and brown dwarf atmospheres. Notably, photometric variability is a common method to constrain the presence and evolution of clouds on an unresolved body, which can play a crucial role in shaping the observed atmospheric spectra.

In the brown dwarf regime, the detection of photometric variability is common. It has been previously demonstrated in large-sample surveys (Girardin et al. 2013; Radigan 2014; Radigan et al. 2014; Wilson et al. 2014; Metchev et al. 2015) that, as suggested by atmosphere models (Showman \& Kaspi 2013), a significant fraction of field brown dwarfs display largeamplitude photometric variations in the infrared, especially at the $\mathrm{L} / \mathrm{T}$ transition. Notable examples in-

*Corresponding author: naud@astro.umontreal.ca clude SIMP J013656.57+093347.3 (SIMP 0136 hereafter), a T2.5 isolated object ${ }^{1}$ that has been shown to display a $J$-band variation up to $6 \%$ peak-to-peak over a period of $2.4 \mathrm{hr}$ (Artigau et al. 2009; Apai et al. 2013; Metchev et al. 2013; Croll et al. 2016), 2MASS J21392676+0220226 (2MASS J2139 hereafter), a T1.5 displaying a peak-to-peak variability as large as $26 \%$ in $J$ band over $7.7 \mathrm{hr}$ (Radigan et al. 2012); and WISE J104915.57-531906.1 (Luhman 16B), a T0.5 that shows a $>10 \%$ peak-to-peak amplitude variability with a $\sim 5 \mathrm{hr}$ period in the near-infrared (Gillon et al. 2013; Burgasser et al. 2014; Buenzli et al. 2015). The cooler T6.5 2MASS J22282889431026 (2M 2228 hereafter; Buenzli et al. 2012) shows rapid variability (period $1.4 \mathrm{hr}$ ) in the near- and mid-infrared bands with peak-to-

\footnotetext{
1 This object, initially thought to be a field brown dwarf, was recently shown to be a likely member of the $\sim 200$ Myr old CarinaNear association, and is thus in all likelihood below the planetarymass threshold of $\sim 13 M_{\text {Jup }}$ (Gagné et al. 2017).
} 
peak amplitudes ranging from $1.45 \%$ to $5.3 \%$. Recently, the Y0 dwarf WISE J140518.39+553421.3 was found to be variable at the $7 \%$ level (peak-to-peak) on a $8.5 \mathrm{hr}$ period, in the Spitzer Infrared Array Camera (IRAC) [3.6] and [4.5] bands (Cushing et al. 2016).

The most common explanation for the observed shortterm variability of brown dwarfs is the presence of a non-uniform cloud cover in the atmosphere (Apai et al. 2013). Doppler imaging allowed us to obtain a twodimensional map of Luhman 16B, the nearest known brown dwarf $(\sim 2 \mathrm{pc})$. It exhibits large-scale bright and dark regions that evolve with time and that naturally explain the observed photometric variability (Crossfield et al. 2014). Fluctuations in the temperature of the atmosphere could provide an alternative explanation for objects outside of the L/T transition (Robinson \& Marley 2014). Variability of 2M 2228 could be explained by a combination of patchy sulfide clouds and hot spots (Morley et al. 2014; Robinson \& Marley 2014). Regardless of the underlying physical mechanism, variability is in all cases primarily produced by modulation due to rotation, which brings regions with different physical properties in and out of sight.

Variability in $\mathrm{T}$ dwarfs was also detected on longer timescales of days to months for most isolated objects studied over such long periods. SIMP 0136 is an extreme example with a peak-to-peak amplitude varying between less than $1 \%$ to more than $6 \%$ over the 6 years it was studied (Artigau et al. 2009; Apai et al. 2013; Metchev et al. 2013; Croll et al. 2016). The evolution of variability is thought to be due to large-scale evolution of weather patterns on the surface. In our solar system, simultaneous disk-integrated and resolved photometric studies of the ice giant Neptune suggested that the short- and long-term evolution of cloud structures on the surface of planets generate variations in the photometric light curves on timescales of hours to months (Simon et al. 2016).

Future instruments on ground-based $30 \mathrm{~m}$ class telescopes and JWST will allow more in-depth photometric variability studies of directly imaged exoplanets (Kostov \& Apai 2012). However, variability studies are currently very challenging to perform on the majority of known giant exoplanets because of the proximity to their host star (e.g., see observations of HR 8799 by Apai et al. 2016). The detection of a photometric modulation in 2MASS J12073346-3932539 b (hereafter 2M 1207 b; Zhou et al. 2016) illustrates that a reliable detection of the rotation-induced modulation can, however, be obtained, at least for the most favorable geometries.

The discovery of free-floating planetary-mass objects allows extending photometric variability studies into the low-gravity regime. In their Spitzer program studying 44 L3-T8 brown dwarfs, Metchev et al. (2015) iden- tified a tentative correlation between low-gravity objects and large-amplitude variability. Biller et al. (2015) found the first evidence for variability for a low-gravity object, PSO J318.5-22, a late-L planetary-mass object and member of the very young $\beta$ Pictoris moving group $(\sim 20 \mathrm{Myr})$. They found the planetary-mass object to be variable with a large amplitude of $7 \%-10 \%$ peakto peak in $J_{S}$ band, at two different epochs. Another low-gravity dwarf, the L6 WISEP J004701.06+680352.1 (W0047 hereafter), was also found to be variable with a very large amplitude in the near-infrared (8\% peakto-peak; Lew et al. 2016). These low-gravity L dwarfs display larger amplitude variability than most variable field L-type brown dwarfs, suggesting that in agreement with Metchev et al. (2015), young, dusty L planetarymass objects could be more variable than their older counterparts of similar colors. The recent finding that the highly variable SIMP 0136 is in all likelihood also young (given its probable membership to the $200 \mathrm{Myr}$ association Carina-Near; Gagné et al. 2017), could suggest that this hypothesis extends for early $\mathrm{T}$ dwarfs. This thus calls for further observations of these young objects.

GU Psc b is a T3.5 \pm 1 planetary-mass companion at a separation of $42^{\prime \prime}$ (2000 au at $48 \mathrm{pc}$ ) from the young M3 star GU Psc, a member of the young ( 150 Myr; Bell et al. 2015) AB Doradus moving group (ABDMG). This very wide companion was identified from its distinctively red $i^{\prime}-z^{\prime}>3.5$ color from the PSYM-WIDE survey carried out on Gemini-South/GMOS (Naud et al. 2017) and confirmed to be co-moving with multi-epoch WIRCam $J$-band astrometry (Naud et al. 2014). Given its estimated $T_{\text {eff }} \sim 1050 \mathrm{~K}$ and the young age inferred from its membership to ABDMG, its estimated mass is at the high-end of the planetary-mass regime $\left(9-13 M_{\mathrm{Jup}}\right)$.

GU Psc b has a similar mass to closer-in giant exoplanets revealed by high-contrast imaging and isolated planetary-mass objects. Besides, it shares similar spectral features to much older and massive field brown dwarfs at the $\mathrm{L} / \mathrm{T}$ transition. Its study allows us to investigate for connections between these two types of objects. As it is one of a few dozen exoplanets that have been directly imaged and one rare case among those that were detected without the aid of adaptive optics, GU Psc b presents an opportunity to study the light curve of an exoplanet similarly to what was done for older early $\mathrm{T}$ dwarfs with current instruments.

Previous observations presented by Naud et al. (2014) showed no $J$-band variability above $150 \mathrm{mmag}$ (at a $3 \sigma$ confidence level) over 3 epochs spanning 11 months. This paper presents the first dedicated monitoring of the broadband photometric variability of GU Psc b. In $\S 2$, observations obtained at the Canada-France-Hawaii Telescope (CFHT) in 2013-2014 are described. The 
light curves obtained are presented in $\S 3$. In $\S 4$, the analysis of these light curves is detailed. Finally, the importance of this result in light of other recent variability studies is discussed in $\S 5$, and future observations that could reveal additional insights on the atmospheric dynamics of planetary-mass objects are suggested.

\section{OBSERVATIONS AND DATA REDUCTION}

Observations of GU Psc b were obtained through Director's discretionary time at the $\mathrm{CFHT}^{2}$ with the nearinfrared camera WIRCam (Puget et al. 2004). Since rotation periods for young low-mass companions are still largely unknown, the longest continuous observation span that could be secured continuously on CFHT was requested. Three $5-6 \mathrm{hr} J$-band observing periods on three different nights were obtained: on 2013 December 22, and on 2014 October 10 and 2014 October 11 (see Table 1). Long exposure times (50 s in 2013 December and $60 \mathrm{~s}$ in 2014 October) were used in order to achieve the best possible signal-to-noise for this faint target $\left(J_{M K O}=18.12\right.$, Naud et al. 2014).

WIRCam is equipped with four $2048 \times 2048$ pixels HAWAII 2RG detectors (pixel scale of $0.3307 /$ pixel) spanning a field of view of $20^{\prime} \times 20^{\prime}$. The target was kept approximately at the same position in an area clean of cosmetic defects in the northwest detector for the complete duration of a given observation epoch, using the WIRCam staring mode (Devost et al. 2010). WIRCam was purposely slightly defocused relative to the best focus position of the primary mirror, just enough to stabilize the PSF in the event of changing seeing conditions while keeping the PSF Gaussian and ensuring that the flux remained significantly above the sky level. The dialed defocus was $0.20 \mathrm{~mm}$ and never drifted more than $0.05 \mathrm{~mm}$ from the telescope model position, which ensured that the PSF had a minimum FWHM of $2.2 \pm 0.5$ pixels. Appropriate master twilight flats and darks were obtained in the standard CFHT calibration sequences. In 2013 December, sky observations were obtained but they did not allow us to improve the quality of the results and were thus not used. No further sky observations were obtained in 2014 October.

The IDL Interpretor of WIRCam Images (I'iwi v2.1.200 ${ }^{3}$ ) was used for preprocessing of the raw data including dark subtraction, flat fielding with twilight flat, bad pixel mapping and nonlinearity correction.

\section{RESULTS}

\subsection{Raw Light Curves}

\footnotetext{
2 Run IDs 13BD91 and 14BD88.

3 See http://www.cfht.hawaii.edu/Instruments/Imaging/ WIRCam/IiwiVersion1Doc.html
}

Table 1. Observation Log

\begin{tabular}{cllc}
\hline \hline $\begin{array}{c}\text { Date } \\
\text { (UTC) }\end{array}$ & Time Start & $\begin{array}{c}t_{\exp } \\
(\mathrm{s})\end{array}$ & $\begin{array}{c}\text { Total Duration } \\
(\mathrm{hr})\end{array}$ \\
\hline 2013 Dec 22 & $04: 41: 22$ & 50.0 & 5 \\
2014 Oct 10 & $8: 40: 37$ & 60.0 & 6 \\
2014 Oct 11 & $7: 07: 20$ & 60.0 & 6 \\
\hline
\end{tabular}

IDL procedures were used to perform aperture photometry on the target for each individual exposure. The same operation was performed on 40 stars identified in the field, located close to the target on the same detector and with a brightness between 0.1 and 10 times that of the target (see Figure 1).

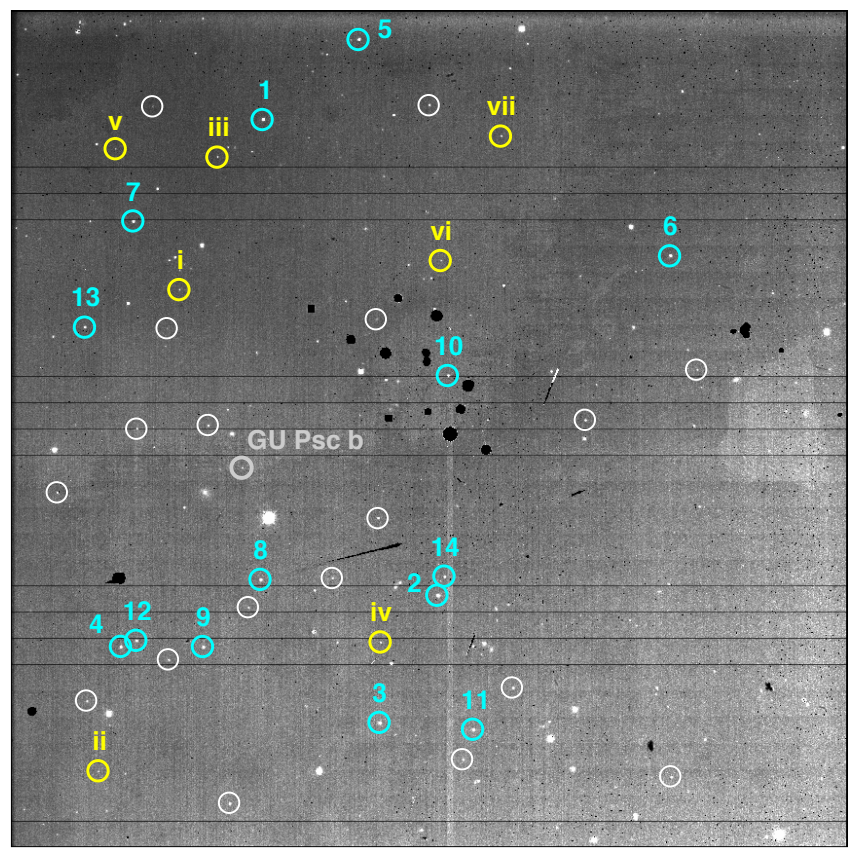

Figure 1. Deep image of the field observed in $J$ band with WIRCam (stack of the $6 \mathrm{hr} 2014$ October 11 epoch). The field shown is the northwest detector of WIRCam (\#77) and is $10^{\prime}$ on a side. GU Psc b and the 40 stars considered in the analysis are circled. Among these stars were selected 14 reference stars, high SNR stars that are in the 2MASS catalog, which were used to correct the light curves. They are the same for the three observation runs. They are identified in cyan and numbered 1-14. Seven comparison stars, with a brightness similar to GU Psc b, used to validate the results, are circled in yellow and numbered i to vii.

The position and FWHM of all stars at each time step were first determined using the IDL procedure MPFIT2DPEAK, which adjusts a 2D Gaussian profile at the approximate position identified manually. The IDL procedure APER was then used to do aperture photometry and extract the raw light curves. An aperture fixed in size (rather than a multiple of the changing FWHM) 
located at the median position was adopted all along the observations of a given epoch. This aperture proved to generate the most stable light curves, even though the precise position of the stars and the FWHM of their PSF varied during the observation (see Figure 2 for 2011 October 11, and Figures 10 in the Appendix for 2013 December 22 and 2014 October 10).

Apertures of various sizes ranging from a radius of 1 to 8 pixels were tested. An aperture of 3 pixels was selected. This aperture is small enough to minimize the Poisson noise for our faint target (sky-background dominated) but large enough to avoid systematic errors due to the loss of flux caused by slight displacements of the star and variations in the seeing at a given epoch and across the field. Annuli with inner and outer sky radii of 4.5 and 9 pixels were used to measure the sky contribution. The measured flux was converted to a relative flux by dividing the entire light curve of every star by its median. The raw light curve generated for GU Psc b on 2014 October 11 is shown in Figure 3. The raw curves for the two other nights are shown in Figure 11 of the Appendix. Among the 40 stars initially identified for which a raw light curve was extracted (all stars circled in Figure 1), seven stars with a brightness similar to that of GU Psc b (85\%-130\% of the flux of the target, identified by yellow circles and roman numerals in Figure 1 ) were selected as comparison stars. Their median raw light curve is also displayed in Figure 3. A set of 14 reference stars were also selected, consisting of bright stars that have a high signal-to-noise ratio (larger than $\sim 60$ per measurement), that are not obviously variable and that are listed in the 2MASS catalog (identified by cyan circles and arabic numerals in Figure 1). Their characteristics are listed in Table 2 and their median raw light curve is shown in Figure 3. The reference stars are used to correct the raw light curves of GU Psc b and comparison stars (see section 4 for more detail on the procedure used to do so).

The variations of several external parameters such as the position on the detector, seeing, airmass and temperature were also monitored to study their possible effect on the data (Figure 2). The variation of positions, which were found to be similar for stars on the same detector (Figure 2 shows the median of all stars), arises partly from the WIRCam science acquisition sequence. At the end of each observing sequence (about $1 \mathrm{hr}$ ), the position of the pointing was adjusted. That can be seen, for example on 2014 October 11 by the variation of the $y$ position every hour. The measured FWHM of the PSF also varied during a given epoch due to the changing seeing. The median FWHM of the PSF is 2.4 pixels for the first night, 2.3 pixels for the second night, and 2.5 pixels for the third. On the first night, an important degradation of the seeing can be seen $\sim 4.5 \mathrm{hr}$ after the beginning of the observations. Observations were obtained at airmasses below 2.0.

\section{ANALYSIS}

\subsection{Principal Component Analysis}

A common procedure to eliminate instrumental noise common to all observed stars is to divide the raw light curves by a reference curve, which is usually built from the mean or median of carefully selected reference stars (Radigan et al. 2014). However, in the present case, such a procedure leaves residual variability that is not likely of astrophysical nature, as patterns can be recognized in the "corrected" light curves of both GU Psc b and comparison stars, and seem correlated with external parameters.

A principal component analysis (PCA; Jolliffe 2002) was used to efficiently eliminate this common instrumental noise. The raw light curves of the 14 reference stars were used as inputs in the IDL function PCA, which computes their covariance matrix and finds its eigenvectors (or Principal Components; PC) and eigenvalues. Principal components, which are orthogonal by construction, are ordered in decreasing contributions to the variance of the sample set. A few PCs usually contribute to most of the variance in the data, as was observed here, hence those with less significance were ignored. A Scree plot was used to determine how many $\mathrm{PC}$ to retain. This plot displays eigenvalues in decreasing order, and only the PCs that have a value in the steep decline, before a plateau is reached, are kept. For the three nights, 3/14 PCs were used, which accounted for more than $98 \%$ of the variance in all three cases. These PCs are displayed in Figure 4 for the third epoch, and in the Appendix (Figure 12) for the two other. It was verified that retaining one more or one less PC does not affect the results significantly.

Comparing the principal components to the variation of external parameters (shown in Figure 2) can shed light on what has the most effect on the observed common variability. The strongest effect is the variation of the seeing. The first PC shows an anti-correlation of $>95 \%$ with the measured FWHM for the three epochs. A few other external parameters have recognizable patterns that can be found in the principal components. The jumps that are seen approximately at 1 and $5 \mathrm{hr}$ in the $\Delta y$ curve of Figure 2 (2014 October 11) can be seen in the second PC. Other varying parameters could have an impact as well, but the effect of the smoothly varying ones (sky level, airmass) are harder to disentangle.

\subsection{Autocorrelation Analysis}

The following procedure was applied to the reference stars to evaluate the presence of correlated noise in the light curves. An optimal reference light curve was built 

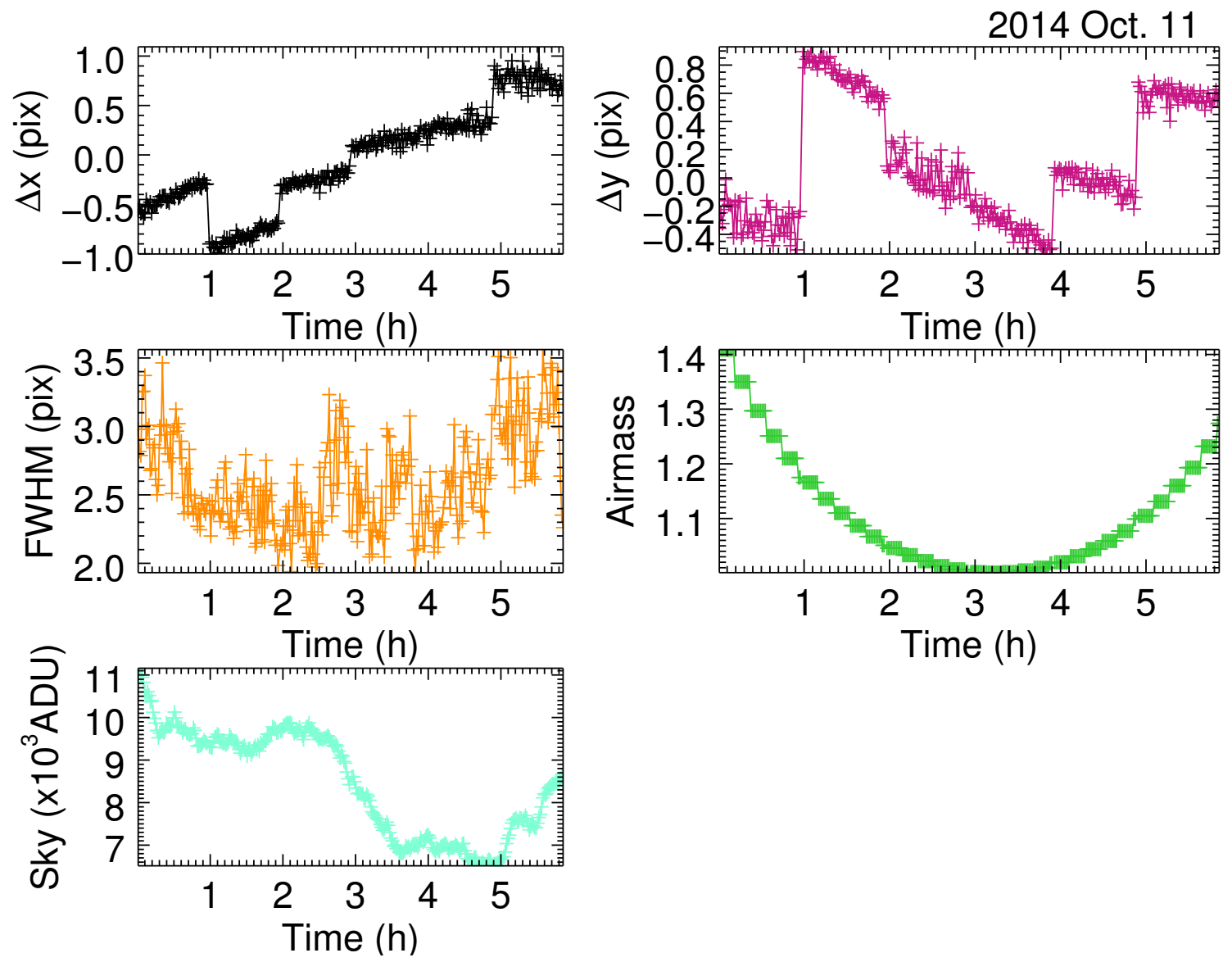

Figure 2. Variation of different external parameters during the 2014 October 11 epoch. The variation of the $x$ and $y$ positions on the chip (median of all stars), FWHM, airmass, and sky level (ADU) are shown. Similar figures for the two other nights are shown in the Appendix.

Table 2. Selected Reference Stars

\begin{tabular}{ccccccc}
\hline \hline 2MASS & ID $^{\mathrm{a}}$ & Distance & R.A. & \multirow{2}{*}{$\begin{array}{c}\text { Decl. } \\
\text { Designation }\end{array}$} & & 2MASS \\
\cline { 5 - 7 } from Target $\left(^{\prime}\right)$ & $(\mathrm{deg})$ & $(\mathrm{deg})$ & $J$ & $K_{s}$ \\
\hline J01123542+1708511 & 1 & 4.33 & 18.148 & 17.148 & 15.514 & 14.5490 \\
J01122636+1702556 & 2 & 2.90 & 18.110 & 17.049 & 15.481 & 14.8010 \\
J01122937+1701205 & 3 & 3.61 & 18.122 & 17.022 & 15.638 & 14.8110 \\
J01123039+1709489 & 5 & 5.48 & 18.127 & 17.164 & 15.852 & 15.2760 \\
J01121425+1707070 & 6 & 5.91 & 18.059 & 17.119 & 15.825 & 15.1590 \\
J01124214+1707359 & 7 & 3.35 & 18.176 & 17.127 & 16.133 & 15.4240 \\
J01123560+1703082 & 8 & 1.41 & 18.148 & 17.052 & 16.099 & 15.2590 \\
J01123859+1702178 & 9 & 2.29 & 18.161 & 17.038 & 16.027 & 15.3530 \\
J01122580+1705389 & 10 & 2.79 & 18.108 & 17.094 & 16.408 & 15.8430 \\
J01122456+1701154 & 11 & 4.34 & 18.102 & 17.021 & 16.520 & 16.1250 \\
J01124208+1702228 & 12 & 2.53 & 18.175 & 17.040 & 16.921 & 15.4570 \\
J01124468+1706169 & 13 & 2.63 & 18.186 & 17.105 & 16.662 & 15.5090 \\
J01122599+1703093 & 14 & 2.86 & 18.108 & 17.053 & 16.333 & 15.4090 \\
\hline
\end{tabular}

Note ${ }^{\text {a }}$ The ID refers to Figure 1 


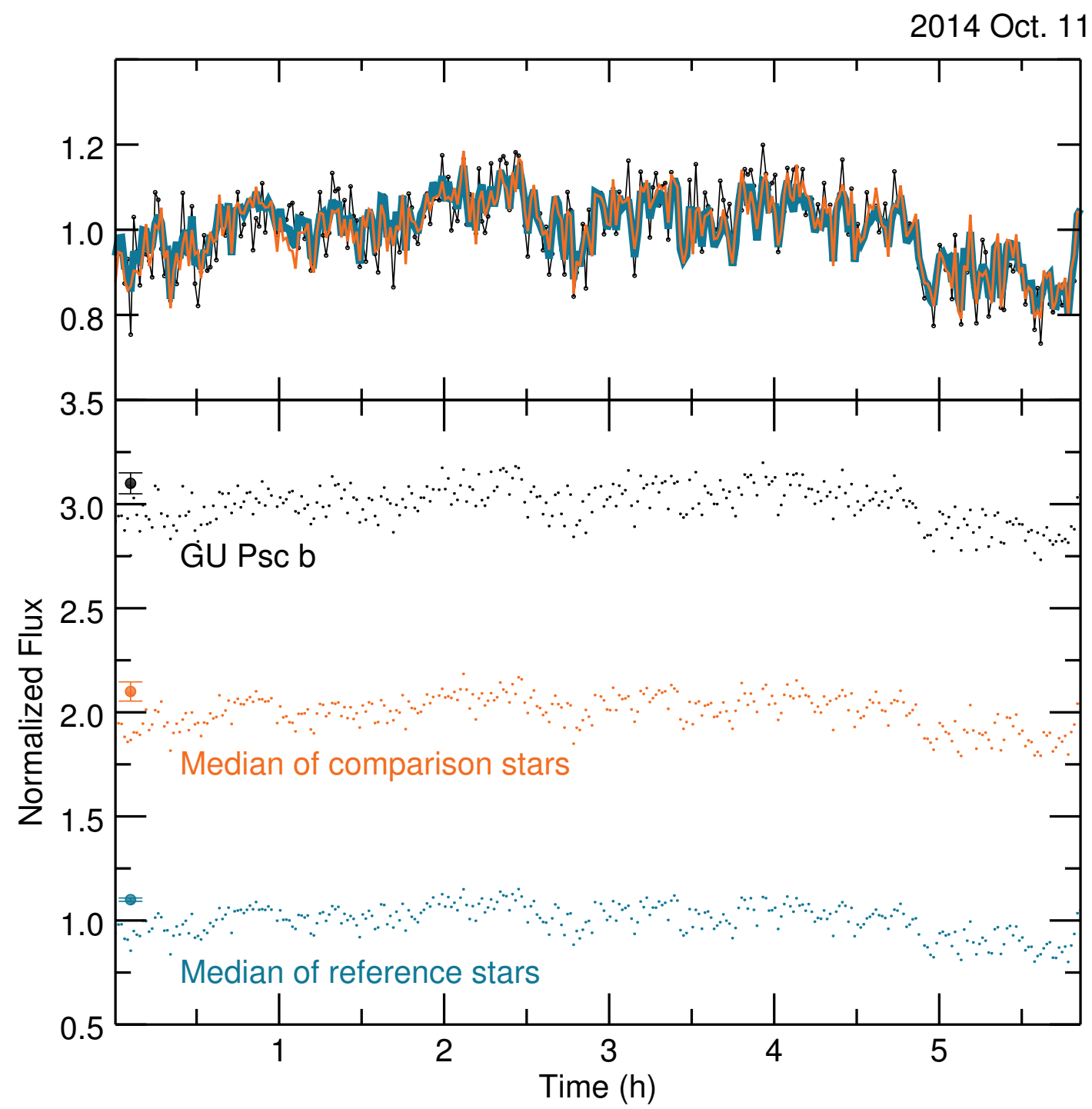

Figure 3. Bottom panel: raw normalized light curves for GU Psc b (top curve, in black), the median of comparison stars (middle curve, in orange) and the median of all reference stars (bottom curve, in cyan), for 2014 October 11. GU Psc b and comparison star median curves have been offset for clarity. Top panel: the same curves, overplotted over one another, GU Psc b is the black curve, with dot symbols, the median of the reference stars is the thick dark cyan line, and the median of the comparison stars is the thin orange pale line. The raw curves for the two other nights are shown in the Appendix.
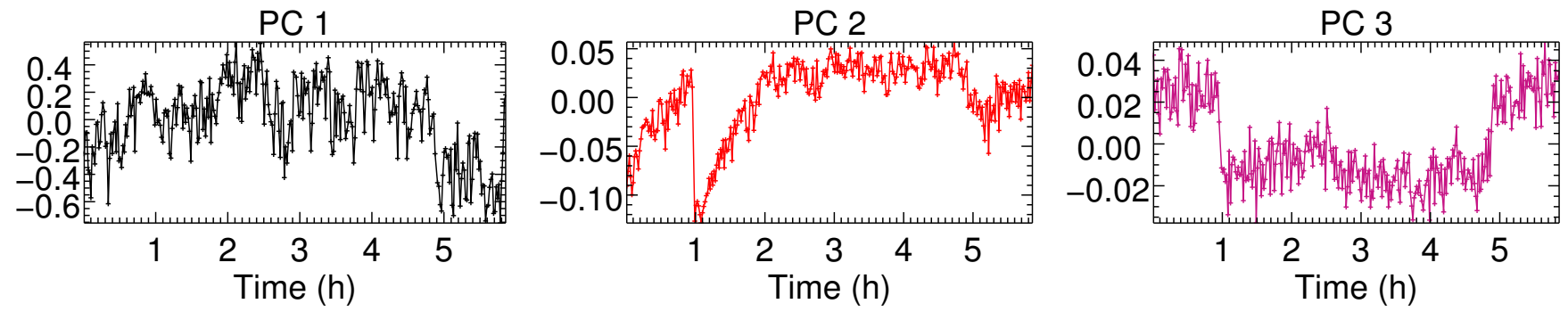

Figure 4. Most important principal components for 2014 October 11, obtained from the light curves of the 14 reference stars (identified with arabic numbers in Figure 1). Similar figures for the two other nights are shown in the Appendix. 
for each of the 14 reference stars using the linear combination of the three first principal components, which minimizes the RMS of residuals in the corrected light curve. In each case, the reference star in question was excluded from the determination of the PC. Corrected light curves were obtained by subtracting the optimal reference light curve from the raw light curves. An autocorrelation analysis was carried out on the residuals of these corrected light curves. Figure 5 shows the autocorrelation curves at all epochs for the median of the 14 stars. The analysis showed no evidence for noise correlation on timescales longer than 30 minutes. For timescales significantly longer than 30 minutes and variability amplitudes larger than the RMS of bright stars ( $0.5 \%$ peak-to-peak), the noise can therefore be assumed to be white.

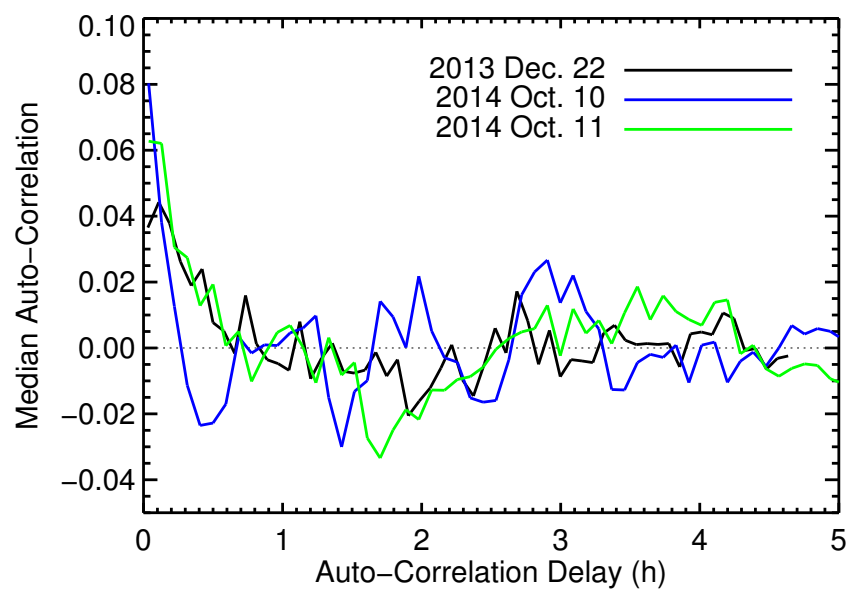

Figure 5. Result of the autocorrelation analysis that was carried out. The reference star light-curve residuals are used, after the removal of the instrumental noise with PCA analysis. The median of the autocorrelation curves of the 14 reference stars is shown for each epoch.

\subsection{Correcting for Instrumental Noise and Variability Modeling}

Instrumental noise and astrophysical variability can often be correlated. A joint model was used to simultaneously find the scale of the PCs (to remove instrumental noise) and find the best fit to a possible intrinsic variability. This procedure allows us to avoid problems caused by a correlation between the two. The model in question is described by

$$
m_{i}=A \cos \left[\frac{2 \pi}{P}\left(t_{i}+t_{0}\right)\right]+\sum_{k=1}^{3} B_{k} \omega_{i k}+C,
$$

where $A$ is the amplitude of intrinsic variability, $P$ is the period, and $t_{0}$ is the time offset. The three principal components (noted $\omega_{i k}$ ) are scaled by $B$. Even if the light curves of many variable brown dwarfs and planetary-mass companions are not sinusoidal, the present model was selected as a simplistic choice to search for stellar variability without overfitting the data. The model is rewritten in the following linear form to allow for a matrix inversion:

$m_{i}=A_{C} \cos \left(\frac{2 \pi}{P} t_{i}\right)+A_{S} \sin \left(\frac{2 \pi}{P} t_{i}\right)+\sum_{k=1}^{3} B_{k} \omega_{i k}+C$

with $A=\sqrt{A_{S}^{2}+A_{C}^{2}}$ and

$$
t_{0}=\frac{P}{2 \pi} \arccos \left(\frac{A_{C}}{\sqrt{A_{C}^{2}+A_{S}^{2}}}\right) .
$$

The optimal parameters $A, P, t_{0}, B_{1}, B_{2}, B_{3}$ and $C$ are determined for periods ranging from $\sim 10$ minutes up to twice the total duration of the observation period $(12 \mathrm{hr})$, in step of 6 minutes. The resulting peak-to-peak amplitude $(2 A)$ for each trial period are shown in Figure 6 for GU Psc b (solid blue line).

A Monte Carlo simulation was performed to evaluate the detection limits: the data points were shuffled randomly 10,000 times, and each new set of data was fitted again, yielding an amplitude for each trial. The $68 \%$, 95\%, and $99.7 \%$ lines on Figure 6 for GU Psc b (dotted, dashed, dash-dotted red lines) represent the amplitudes below which $68 \%, 95 \%$, and $99.7 \%$ of the simulations are found.

A $\sim 4-5 \%$ peak-to-peak amplitude signal is detected at a period of $\sim 6 \mathrm{hr}$ from the analysis for the third epoch. No significant variability is detected for GU Psc b on the first two epochs. A similar analysis for the comparison stars was also carried out. Five of the seven stars show no variability at this level of significance on the three epochs. Star v shows a $>3 \sigma$ variation on the same date, around a period of $4.7 \mathrm{hr}$ and with a peak-to-peak amplitude of $4 \%$, and a $\sim 3 \sigma$ variation on 2014 October 10, with a period and an amplitude that are a bit smaller $(3.9 \mathrm{hr}, 2.7 \%)$. Star i shows a $>3 \sigma$ variation at the third epoch as well, with a period of $2.7 \mathrm{hr}$ and a peak-to-peak amplitude of $2.5 \%$.

\subsection{Monte Carlo Markov Chain Analysis}

A Monte Carlo Markov Chain (MCMC) analysis was carried out on the GU Psc b 2014 October 11 light curve to assess confidence in the detection of variability and explore the parameter space and possible correlations between them. The model shown in equation 1 was used. Since the autocorrelation analysis showed no correlated noise, an uncorrelated Gaussian noise likelihood function was adopted, given by

$$
\log \mathcal{L}=-\frac{1}{2} n \log (2 \pi)-n \log \sigma-\frac{1}{2} \sum_{i=1}^{n}\left(\frac{d_{i}-m_{i}}{\sigma}\right)^{2},
$$



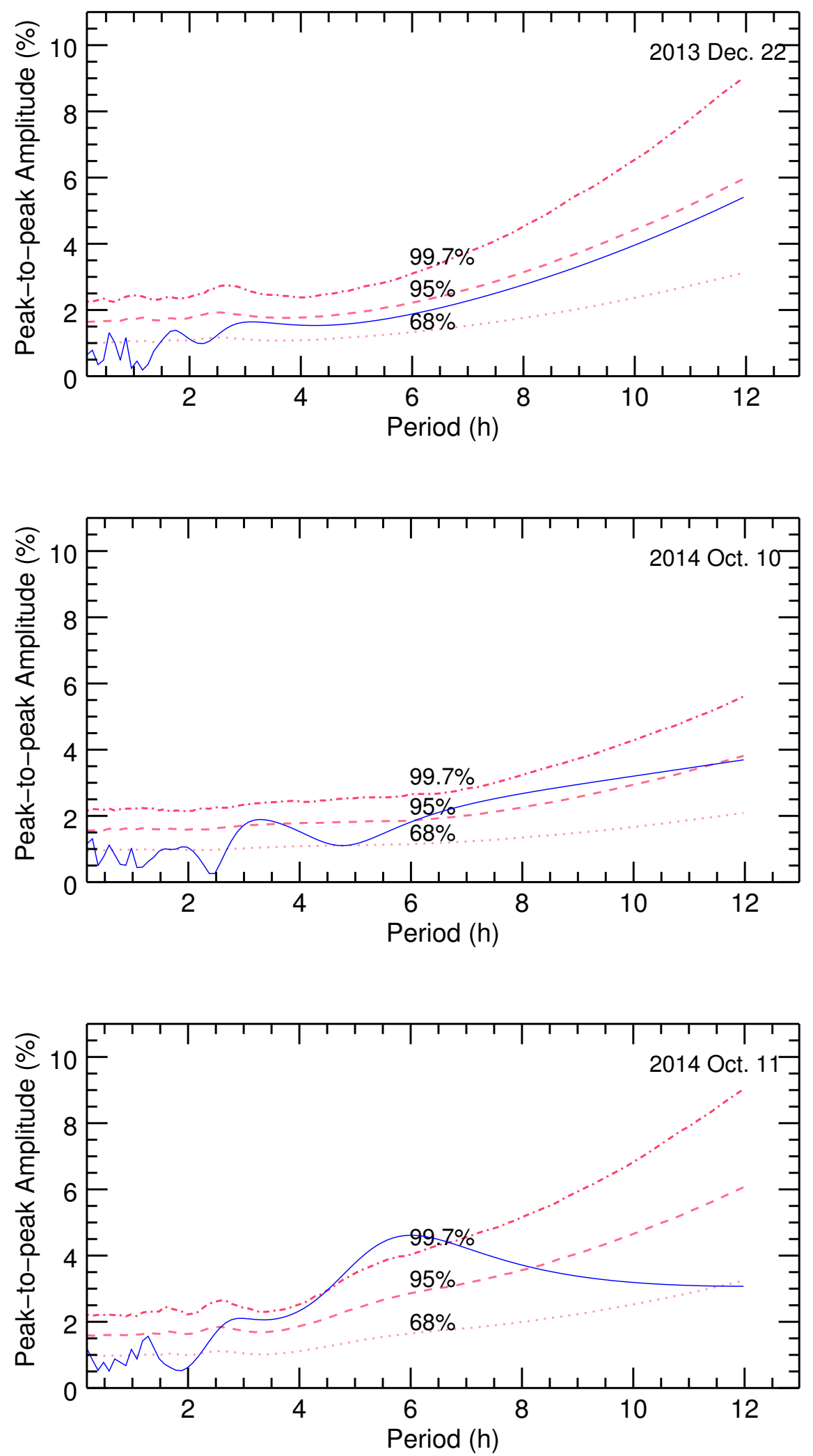

Figure 6. Amplitude of the best-fit model for all periods tested on the light curve of GU Psc b (solid line), for the three epochs. We use the unbinned data in the fit and simulation. The $68 \%, 95 \%$, and $99.7 \%$ detection limits are also shown (dotted, dashed, dash-dotted lines). No significant variability is detected (above the $99.7 \%$ limit) for the first and second nights. On the third night, a $4-5 \%$ peak-to-peak amplitude is seen around $6 \mathrm{hr}$, just above the $99.7 \%$ limit. 
where $n$ is the number of observations, $d_{i}$ the photometric measurements, and $m_{i}$ is the model as given by Equation 1. An uncertainty $\sigma$, that is assumed constant through the entire observation period, was left as a free parameter in the model fits. The model has eight free parameters: $A, P, t_{0}, B_{1}, B_{2}, B_{3}, C$ and $\sigma$. Uniform priors were adopted but restrictions were applied: $A$ was forced to be positive, $P$ between 0 and $12 \mathrm{hr}, t_{0}$ between -10 and $20 \mathrm{hr}, B_{k}$, and $C$ forced to be between -10 and 10 and $\sigma$ was forced to be positive. The MCMC routine of Rowe et al. (2014) was adopted for the analysis. It uses the Metropolis-Hastings algorithm with a hybrid Gibbs and DE-MCMC sampler to efficiently handle correlated variables as described in Gregory (2010).

Three chains (sequences of "states" that have a given value for each parameter) with lengths of 50,000 were generated. A visual examination of the chains showed good mixture and the Gelman-Rubin convergence criteria yielded $R_{c}=1.01$ or lower for all fitted parameters (a $R_{c}<1.1$ is a good indicator that convergence was reached; Gelman \& Rubin 1992; Brooks \& Gelman 1998). Figure 7 displays histograms of the chain values for each parameter and scatter plots to unveil potential correlations between various parameters for GU Psc b on 2014 October 11. While it is apparent from Figure 7 that some parameters show some degree of correlation (e.g. $B_{2}$ and $A$ ), overall each parameter is characterized by a well-defined, peaked distribution. This shows that parameters are well bound given the light-curve model adopted in our analysis. From this analysis, the variability of GU Psc b, as observed at this epoch, can be described by a cosine with a peak-to-peak amplitude of $4 \pm 1 \%$ at a timescale of $\sim 6 \mathrm{hr}$. The MCMC calculations show that the posterior distribution for the period has a long tail toward large values, with larger periods becoming increasingly unlikely. At periods much longer than the span of observations, the cosine becomes increasingly degenerate with a straight line. The upper limit was increased from 12 to $20 \mathrm{hr}$ and that did not change the $1 \sigma$ uncertainties. However, it does demonstrate that it is not possible from the current observations to determine the true periodicity of the variability of GU Psc b. The mode and $1 \sigma(68.27 \%)$ confidence intervals for all model parameters are reported in Table 3. This result is compatible to what was obtained in subsection 4.3.

The MCMC routine was repeated for the two other epochs and each of the seven comparison stars for all epochs. Median values of peak-to-peak amplitudes $(2 A)$ ranges from $1 \%$ to $4 \%$, where that of GU Psc b at the third epoch is the largest.

Figure 8 shows, for GU Psc b and all comparison stars, the raw light curves that have been corrected using the linear combination of the PCs with the median $B_{k}$ coefficients from the MCMC analysis. Random states of
Table 3. Model Parameters for GU Psc b on 2014 October 11 from MCMC analysis

\begin{tabular}{lccc}
\hline \hline Parameter & Median & $+1 \sigma$ & $-1 \sigma$ \\
\hline $2 A(\%)$ & 4 & +1 & -1 \\
$P$ (hours) & 5.9 & +0.7 & -0.7 \\
$t_{0}$ (hours) & 4.1 & +0.4 & -0.2 \\
$B_{1}$ & 0.30 & +0.02 & -0.01 \\
$B_{2}$ & -0.2 & +0.1 & -0.1 \\
$B_{3}$ & -0.2 & +0.2 & -0.2 \\
$C$ & 1.000 & +0.002 & -0.003 \\
$\sigma$ & 0.040 & +0.002 & -0.002 \\
\hline
\end{tabular}

the MCMC chains for the cosine model are overplotted in red. For GU Psc b, a significant signal can be seen by eye on the corrected light curve for 2014 October 11 . A similar amplitude, albeit of longer period also seems visible on 2014 October 10, but this is not statistically significant. The light curves of most comparison stars appear less variable, by such an inspection. A notable exception is the third epoch of star v.

\subsection{Bayesian Information Criterion}

The maximum likelihood for two models was computed on the light curves of GU Psc b and the comparison stars in order to quantitatively assess the significance of the detection for GU Psc b at the third epoch. Model 1, the cosine model, used Equation 1 with $A$ fixed to the median value from the MCMC analysis ( 7 degrees of freedom, DOF). Model 2 had $A, P$ and $t_{0}$ fixed to zero, which is equivalent to fitting a flat line (a non-variable model) to the data simultaneously with principle components (5 DOF). The maximum likelihood was found using the L-BFGS-B code (Zhu et al. 1997). This code is a limited memory, quasi-Newton method that approximates the Broyden-Fletcher-Goldfarb-Shanno algorithm (Press et al. 1992). The Bayesian Information Criterion (BIC) was then computed for each star and each epoch with $n$ data points with 7 DOF for model 1 and 5 DOF for model 2. The BIC penalizes model 1 for additional DOF. The difference between models 1 and 2 was computed. Figure 9 shows the $\Delta \mathrm{BIC}=\mathrm{BIC}_{1}-\mathrm{BIC}_{2}$ versus amplitude for all stars at each epoch. Lower relative values of the BIC indicate a preferred model. According to Kass \& Raftery (1995), a $|\Delta \mathrm{BIC}|$ between 6 and 10 indicate that one model is "strongly" favored over the other, while values above 10 means the best model is "very strongly" favored over the other. By far, the greatest $|\Delta \mathrm{BIC}|(10.2)$ is found for GU Psc b on the third epoch, which means the cosine (variable) model with a peak-to-peak amplitude of $4.2 \%$ is strongly preferred over the flat line (non-variable) in that case. No 


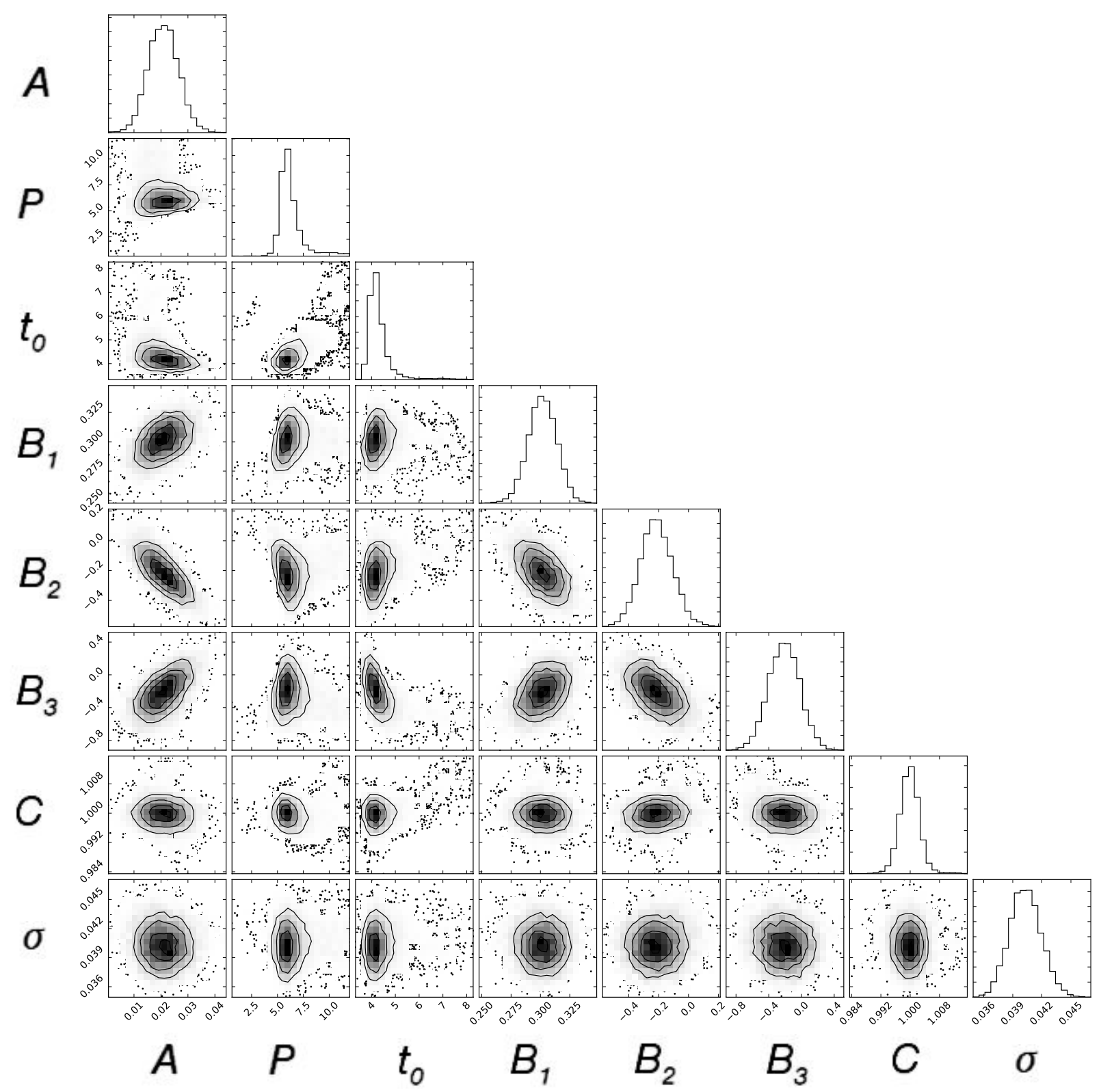

Figure 7. Distributions of parameters (the amplitude $A$, the period $P$, the time offset $t_{0}$, the scale of the three principal components $B_{k}$, the constant $C$ and the constant uncertainty $\sigma$ ) from the MCMC analysis of GU Psc b light curve on 2014 October 11. Scatter plots are produced for each pair of parameters and are a good diagnostic to reveal correlations between model parameters. For example, the amplitude $A$ is correlated with $B_{2}$. Histograms of the parameter distributions are also shown. They provide for each parameter a representation of the posterior distribution.

other star/epoch shows a variability of similar amplitude at this significance level.

\section{DISCUSSION}

The high-precision photometry observations presented here suggest that GU Psc b, a young planetary-mass companion at the cool-end of the $\mathrm{L} / \mathrm{T}$ transition, shows tentative variability on one of the three epochs in which it was observed (2014 October 11). The MCMC analysis suggests a variability with peak-to-peak amplitude of $4 \pm 1 \%$ on a timescale of $\sim 6 \mathrm{hr}$. If confirmed, this would be among the first variability measurements of a planetary-mass companion, GU Psc b being a rare example that is amenable to such high-precision measurements. The observed variability patterns (amplitude, timescale, secular variability) of GU Psc b appear consistent with those observed for brown dwarfs and planetary-mass objects. In the MCMC analysis, at least one of the seven comparison stars, star v, seems to vary significantly at the same epoch, on a shorter timescale and with a slightly lower amplitude. According to the BIC criterion, in that case, the cosine model 


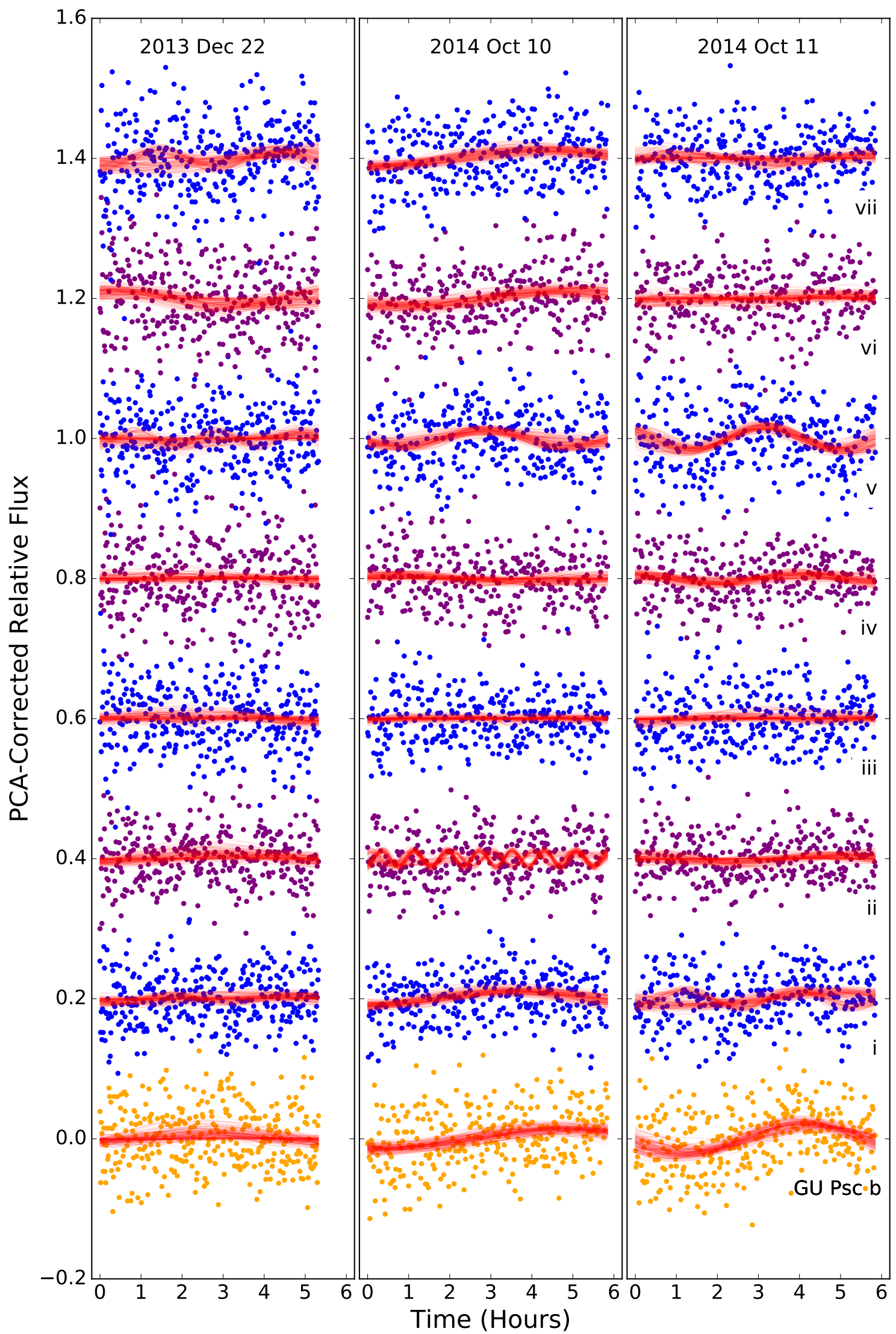

Figure 8. Corrected light curve for GU Psc b and the 7 comparison stars, for the three epochs. Common instrumental noise was removed using PCs with coefficients $B_{k}$ based on the median MCMC values (yellow points for GU Psc b, blue and red points for comparison stars). Two hundred random states of the parameters of the cosine model are shown, overplotted in red, for each light curve. The $\sim 6 \mathrm{hr}$ signal can be seen readily for this corrected light curve for GU Psc b on 2014 October 11. 


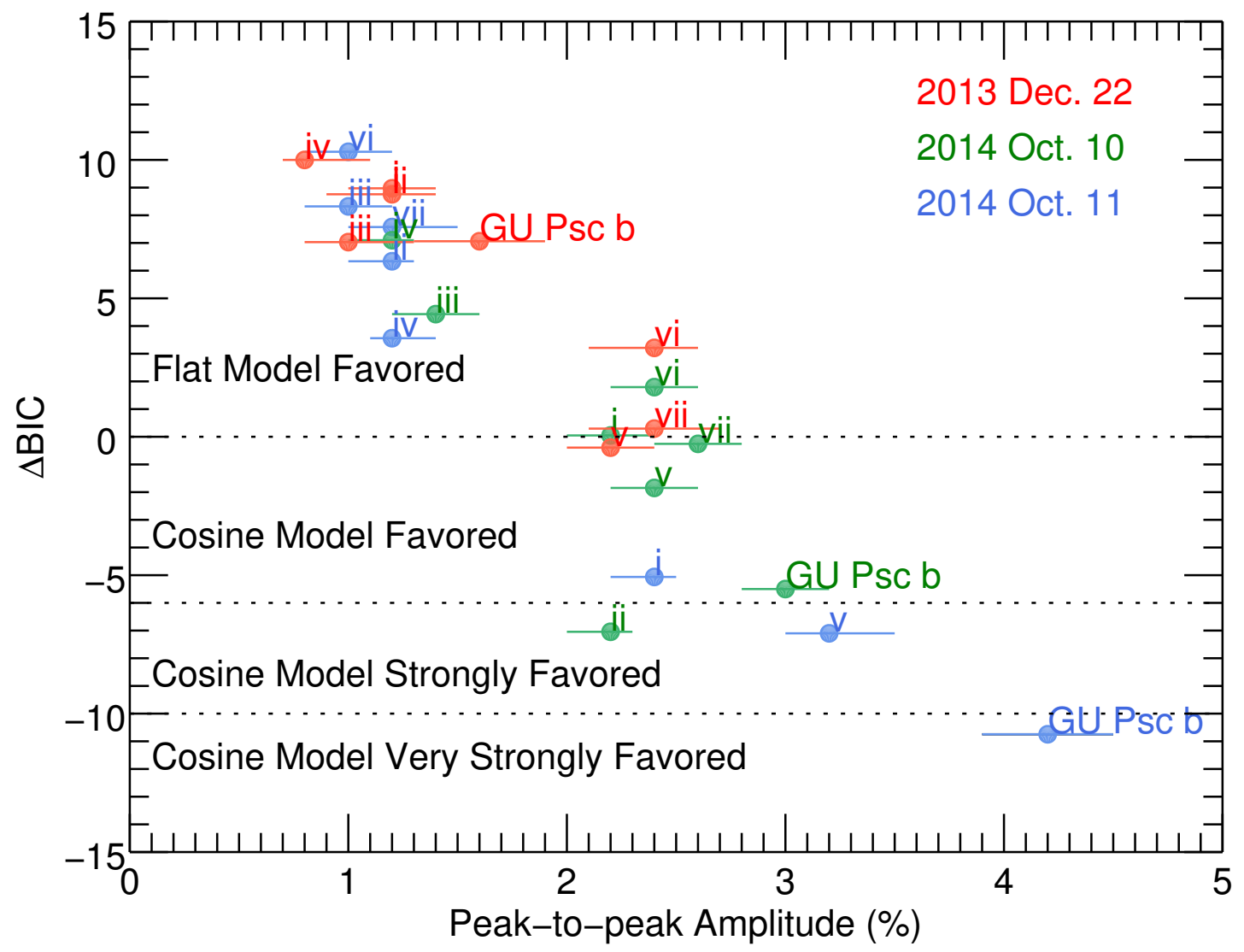

Figure 9. Result of the BIC analysis, which compares the best cosine model and a flat model for GU Psc b and the 7 comparison stars, for the three epochs versus the peak-to-peak amplitude of the cosine model considered. The $\Delta \mathrm{BIC}$ of GU Psc b on the third epoch indicates that the cosine model is below -10 , which means it is very strongly favored over the flat model, according to the Kass \& Raftery (1995) scale. 
is also favored over the straight line. The comparison stars, being randomly drawn in the field, are in all likelihood late-G, $\mathrm{K}$ or early $\mathrm{M}$ stars. Their $i-z, i-J$, $z-J$, and $J-K$ colors, measured in WIRCam (Naud et al. 2014) and SDSS data, are consistent with that. It is thus plausible that the observed variability for comparison star $\mathrm{v}$ is also due to stellar variability (see, e.g., McQuillan et al. 2014).

Based on their Spitzer survey of 44 field and young brown dwarfs, Metchev et al. (2015) confirmed that variability is widespread among $\mathrm{L}$ and $\mathrm{T}$ dwarfs, supporting the findings of Buenzli et al. (2014). They found that $31 \%$ of their single $\mathrm{T} 0-\mathrm{T} 8$ were variable with peakto-peak amplitude between $0.2 \%$ and $4.6 \%$, hence the amplitude suggested here for GU Psc b is expected if the mechanism at play in brown dwarfs extends to the planetary-mass regime. Radigan et al. (2014) and Radigan (2014) argue that strong variability is even more prevalent for the $\mathrm{L} / \mathrm{T}$ transition brown dwarfs, at the limit of which GU Psc b lies.

It is not clear if young objects (often planetary-mass companions) display a similar variability to single field brown dwarfs. Metchev et al. (2015) suggested that in the L dwarf regime, the variable low-gravity Ls could have a higher variability amplitude than the variable field L dwarfs. In accordance with this finding, most of the field L dwarfs studied in the $J$ band by Radigan et al. (2014) are not variable above the $\sim 2 \%$ level. In contrast, the young, late-L, free-floating planetary-mass object PSO 318.5-22 was discovered to be variable with an amplitude of $7-10 \%$ in the $J$ band (Biller et al. 2015). Another young planetary-mass companion, 2M $1207 \mathrm{~b}$, a mid = to late-L-type member of the TW Hya Association ( $8 \mathrm{Myr}$; Gizis 2002; Chauvin et al. 2004), was also recently found to be variable in the near-infrared with an amplitude of $1.36 \%$ in the HST F125W (similar to $J$ band) and $0.78 \%$ in the F160W filters (similar to $H$ band; Zhou et al. 2016), as was the dusty L6 dwarf W0047, which was found to be variable with a peakto-peak amplitude of $8 \%$ with the WFC3s G141 grism, which covers 1.075-1.7 $\mu \mathrm{m}$ (Lew et al. 2016).

In the T-dwarf regime, Metchev et al. (2015) did not find an increased variability frequency or variability amplitude for young $\mathrm{T}$ dwarfs. They studied in the midinfrared two planetary-mass companions that bear some similarities to GU Psc b. The T2.5 HN Peg B, near the deuterium-burning limit, was found to be variable with amplitudes of $0.77 \%$ and $1.1 \%$ in the [3.4] and [4.5] filters, respectively, while the late $\mathrm{T}$ dwarf Ross 458 $(\mathrm{AB}) \mathrm{c}$ showed no variability above $1.4 \%$ and $0.7 \%$ in the same filters. Both companions are somewhat older than GU Psc b but do not have ages that are very well constrained (HN Peg B, $300 \pm 200 \mathrm{Myr}$; Luhman et al. 2007; Leggett et al. 2008, Ross 458 (AB) c, 150-800 Myr,
Burgasser et al. 2010). It could, however, be expected that the variability in the mid-infrared is smaller in amplitude than the variability in $J$ band, where clouds of different temperatures and the atmosphere are expected to show the greatest contrast (Ackerman \& Marley 2001; Marley et al. 2002). For example, with simultaneous near-infrared and Spitzer mid-infrared observations, Yang et al. (2016) showed that the amplitude in $J$ band is two to three times higher than in the Spitzer wavelength for SIMP $0136+0933$. This could thus suggest that HN Peg B would show variability in the $J$ band with an amplitude similar to that measured here for GU Psc b. The fact that the T2.5 SIMP 0136+0933 has probably itself an age that is similar to GU Psc b (around $\sim 200 \mathrm{Myr}$ according to its plausible membership in Carina-Near) is also interesting, since it is one of the most variable objects known.

No high-significance periodic variability was detected for the first two epochs, which were taken approximately 10 months and 1 day before the third epoch. Largescale evolution of weather patterns are suspected to cause long-term changes in the light curve, which may explain these findings. Most brown dwarfs monitored to date show an evolution of their light curve. The 6 yr-monitoring of SIMP 0136 showed that its peak-topeak amplitude varied between $\sim 2 \%$ to more than $10 \%$ (Metchev et al. 2013; Croll et al. 2016). Observations of 2MASS J2139 also suggest an evolution of its light curve over periods of several weeks (Radigan et al. 2012). Neptune, in the solar system, also shows a secular variability (Simon et al. 2016). The fact that the variability observed on 2014 October 11 is not seen on 2014 October 10 with the same significance is also something that can be expected. Metchev et al. (2015) found that many of their variable brown dwarfs showed an evolution of their light curve over timescales of hours only. The T1 Luhman 16B also displays an important evolution from night to night in the 12-day monitoring made with the TRAPPIST telescope (Gillon et al. 2013).

The likely variability detected here could be explained by rotation bringing features in and out of view. Brown dwarfs are known to have relatively short rotation periods, between 2 and $\sim 20 \mathrm{hr}$ (Metchev et al. 2015). The Metchev et al. (2015) survey measured a period of $18 \mathrm{hr}$ for HN Peg B. GU Psc b could have a longer period because it is younger and thus has an inflated radius. For giant exoplanets, rotation periods are still largely unknown. Recently, near-infrared high-resolution spectroscopic observations of Snellen et al. (2014) allowed us to measure an equatorial rotation velocity of $V_{\text {spin }}=25 \mathrm{~km}$ $\mathrm{s}^{-1}$ for the $7 M_{\text {Jup }}$ exoplanet $\beta$ Pictoris b. They assumed that it has a 1.65 Jupiter radius, given that it is a member of the young association $\beta$ Pictoris $(<25 \mathrm{Myr}$, Bonnefoy et al. 2013; Currie et al. 2013; Binks \& Jeffries 
2014; Malo et al. 2014). They estimate a rotation period of about $8 \mathrm{hr}$. In another young association (TW Hya), the young planetary-mass companion 2M1207b was recently observed to vary with a period of $10.7 \mathrm{hr}$ (Zhou et al. 2016) and the free-floating planetary-mass object PSO 318 allowed to constrain its period to $>5 \mathrm{hr}$ (Biller et al. 2015). A period greater than $\sim 6 \mathrm{hr}$ for GU Psc b would thus not be surprising.

Longer observations will be needed to confirm the variability of GU Psc b and its periodic nature, and to eventually better constrain the rotation period. GU Psc b is a prime target for long-term high-precision photometry observation on $8 \mathrm{~m}$ class telescopes, Spitzer and JWST. As GU Psc b seems to show variability in the $J$ band, it would also be interesting to search for variability in other near-infrared or mid-infrared bands, as simultaneous observations in different bands allow to probe different layers of the atmosphere (Buenzli et al. 2012; Apai et al. 2013; Biller et al. 2013; Yang et al. 2016). GU Psc b has a $W 2=15.41$ (Naud et al. 2014), so according to the typical performances achieved with Spitzer in Metchev et al. (2015; see Figure 7), this instrument would be able to detect a variability amplitude down to about $3-4 \%$ in the [4.5] band.

\section{ACKNOWLEDGMENTS}

The authors would like to thank the CFHT staff for their precious help throughout this project. They would also like to thank the anonymous referee and AAS statistics editors for constructive comments and suggestions that improved the overall quality of the paper. This work was financially supported by the Natural Sciences and Engineering Research Council (NSERC) of Canada and the Fond de Recherche Québécois - Nature et Technologie (FRQNT; Québec). Based on observations obtained at CFHT with WIRCam. This publication makes use of data products from the Two Micron All Sky Survey, which is a joint project of the University of Massachusetts and the Infrared Processing and Analysis Center, and funded by the National Aeronautics and Space Administration and the National Science Foundation, of the NASA's Astrophysics Data System Bibliographic Services, SIMBAD database, the VizieR catalog access tool and the SIMBAD database operated at CDS, Strasbourg, France.

Software: I'iwi v2.1.200; IDL MPFIT2DPEAK, APER, PCA

\section{APPENDIX: ADDITIONAL FIGURES}

Raw light curves, external parameter evolution, and retained principal components for the first and second epochs are shown in the Figures 10, 11 and 12.

\section{REFERENCES}

Ackerman, A. S., \& Marley, M. S. 2001, ApJ, 556, 872

Apai, D., Radigan, J., Buenzli, E., et al. 2013, ApJ, 768, 121

Apai, D., Kasper, M., Skemer, A., et al. 2016, ApJ, 820, 40

Artigau, É., Bouchard, S., Doyon, R., \& Lafrenière, D. 2009, ApJ, 701, 1534

Bell, C. P. M., Mamajek, E. E., \& Naylor, T. 2015, MNRAS, 454, 593

Biller, B. A., Crossfield, I. J. M., Mancini, L., et al. 2013, ApJL, 778, L10

Biller, B. A., Vos, J., Bonavita, M., et al. 2015, ApJL, 813, L23

Binks, A. S., \& Jeffries, R. D. 2014, MNRAS, 438, L11

Bonnefoy, M., Boccaletti, A., Lagrange, A. M., et al. 2013, A\&A, 555, A107

Brooks, S. P., \& Gelman, A. 1998, Journal of computational and graphical, 7, 4, 434455 ...

Buenzli, E., Apai, D., Radigan, J., Reid, I. N., \& Flateau, D. 2014, ApJ, 782, 77

Buenzli, E., Saumon, D., Marley, M. S., et al. 2015, ApJ, 798, 127

Buenzli, E., Apai, D., Morley, C. V., et al. 2012, ApJ, 760, L31

Burgasser, A. J., Simcoe, R. A., Bochanski, J. J., et al. 2010, ApJ, 725, 1405

Burgasser, A. J., Gillon, M., Faherty, J. K., et al. 2014, ApJ, 785,48

Chauvin, G., Lagrange, A. M., Dumas, C., et al. 2004, A\&A, 425, L29

Croll, B., Muirhead, P. S., Lichtman, J., et al. 2016, 1609.03587

Crossfield, I. J. M., Biller, B. A., Schlieder, J. E., et al. 2014, Nature, 505, 654
Currie, T., Burrows, A. S., Madhusudhan, N., et al. 2013, ApJ, 776,15

Cushing, M. C., Hardegree-Ullman, K. K., Trucks, J. L., et al. 2016, ApJ, 823, 152

Devost, D., Albert, L., Teeple, D., \& Croll, B. 2010, in SPIE Astronomical Telescopes and Instrumentation: Observational Frontiers of Astronomy for the New Decade, ed. D. R. Silva, A. B. Peck, \& B. T. Soifer (SPIE), 77372D

Gagné, J., Faherty, J. K., Burgasser, A. J., et al. 2017, ApJ, 841, L1

Gelman, A., \& Rubin, D. B. 1992, Statistical science

Gillon, M., Triaud, A. H. M. J., Jehin, E., et al. 2013, A\&A, 555, L5

Girardin, F., Artigau, É., \& Doyon, R. 2013, ApJ, 767, 61

Gizis, J. E. 2002, ApJ, 575, 484

Gregory, P. C. 2010, MNRAS, 410, 94

Jolliffe, I. T. 2002, Principal Component Analysis, second edition edn., Springer Series in Statistics (New York: Springer-Verlag)

Kass, R. E., \& Raftery, A. E. 1995, J. Am. Stat. Assoc., 90, 430, 773

Kostov, V., \& Apai, D. 2012, ApJ, 762, 47

Leggett, S. K., Saumon, D., Albert, L., et al. 2008, ApJ, 682, 1256

Lew, B. W. P., Apai, D., Zhou, Y., et al. 2016, ApJL, 829, L32

Luhman, K. L., Patten, B. M., Marengo, M., et al. 2007, ApJ, 654,570

Malo, L., Doyon, R., Feiden, G. A., et al. 2014, ApJ, 792, 37

Marley, M. S., Seager, S., Saumon, D., et al. 2002, ApJ, 568, 335 

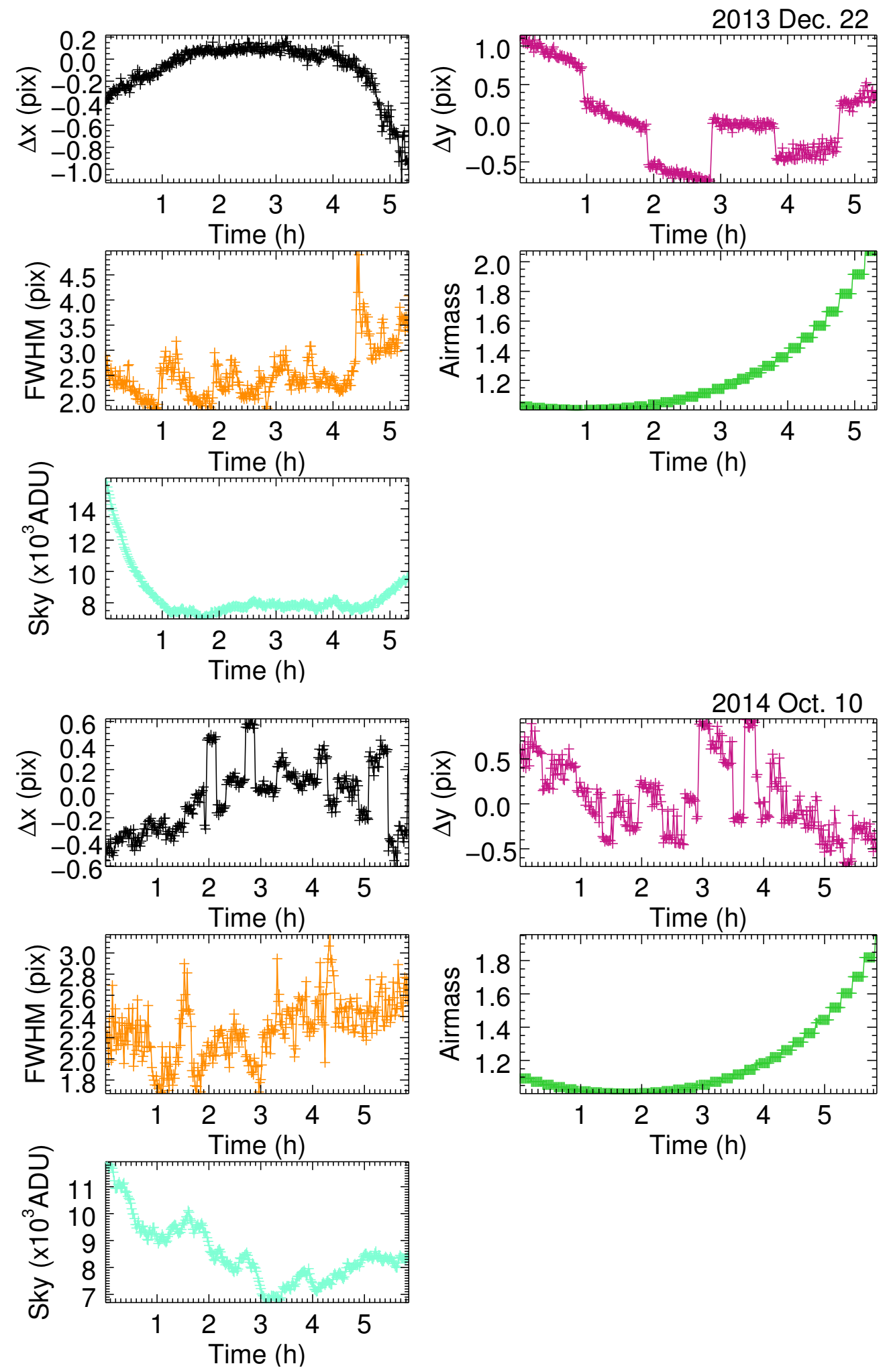

Figure 10. Variation of different external parameters for 2013 December 22 (top) and 2014 October 10 (bottom). The variation of the $\mathrm{x}$ and y positions on the chip (median of all stars), FWHM, airmass, and sky level (ADU) are shown. 

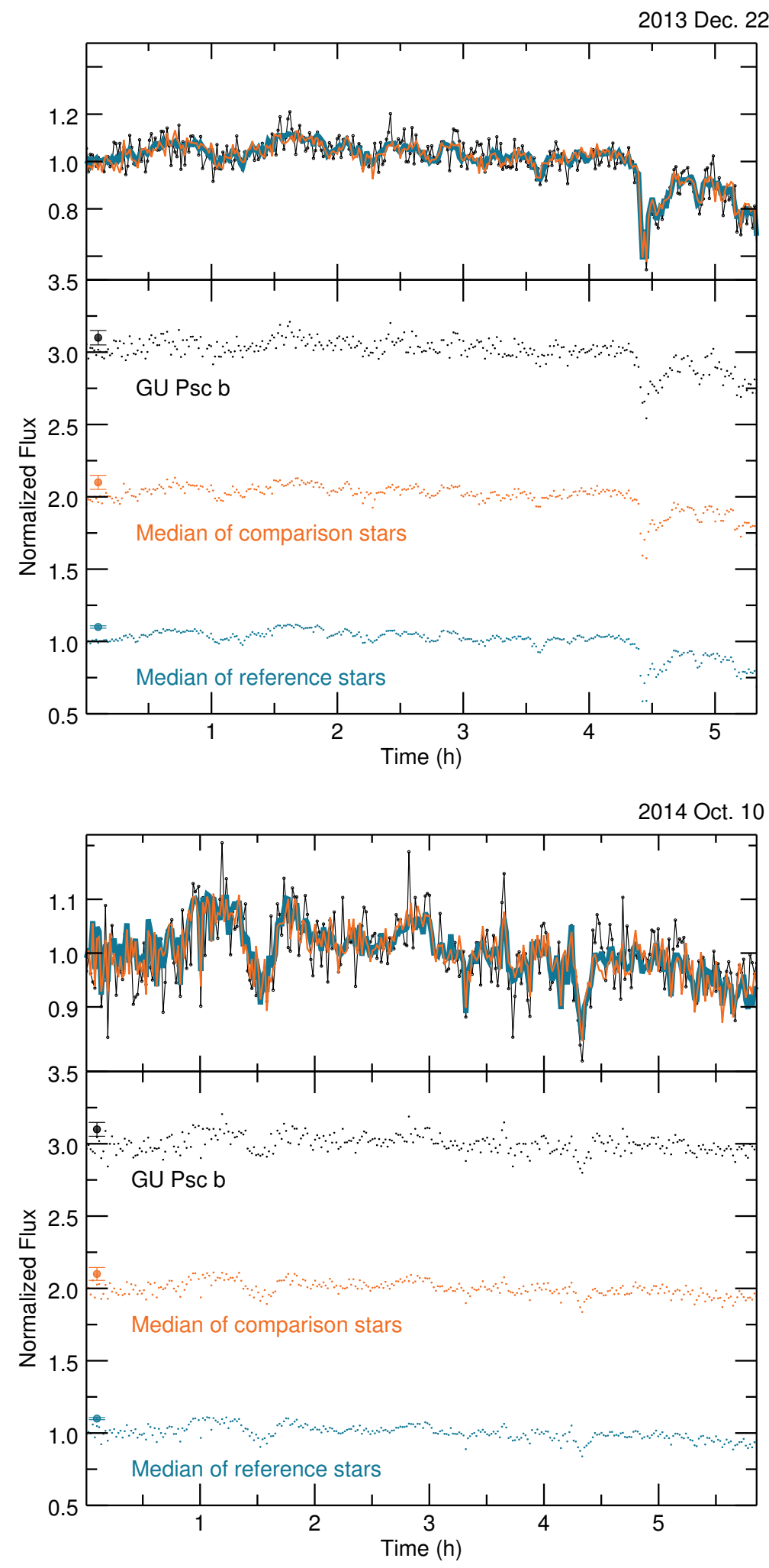

Figure 11. Raw normalized light curves for GU Psc b (top curve), the median of comparison stars (middle) and the median of all reference stars (bottom), for 2013 December 22 (top) and 2014 October 10 (bottom). GU Psc b and comparison star median curves have been offset for clarity. For each date, a superposition of the three curves is also shown above the three curves. GU Psc b is the black curve, with dot symbols, the median of the reference stars is the thick dark cyan line, and the median of the comparison stars is the thin orange pale line. 

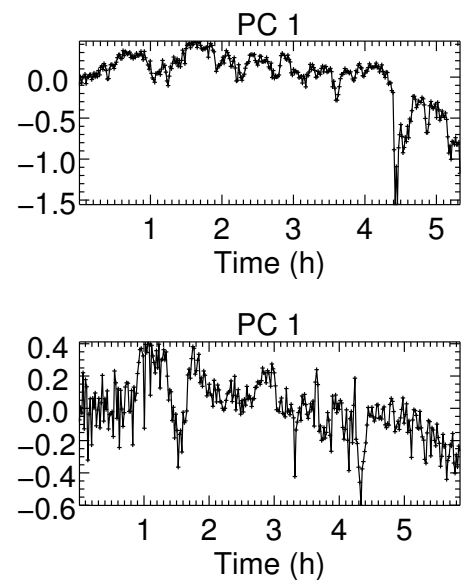
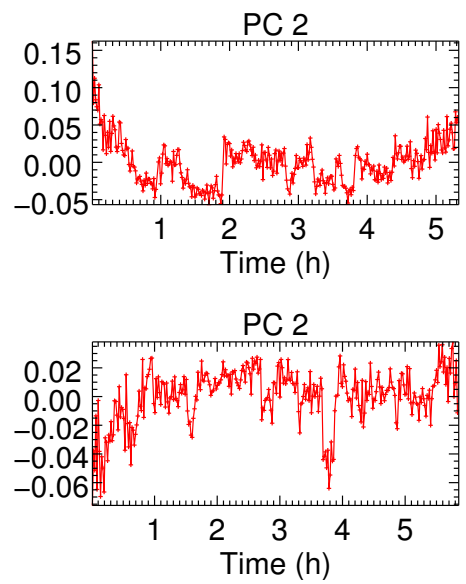
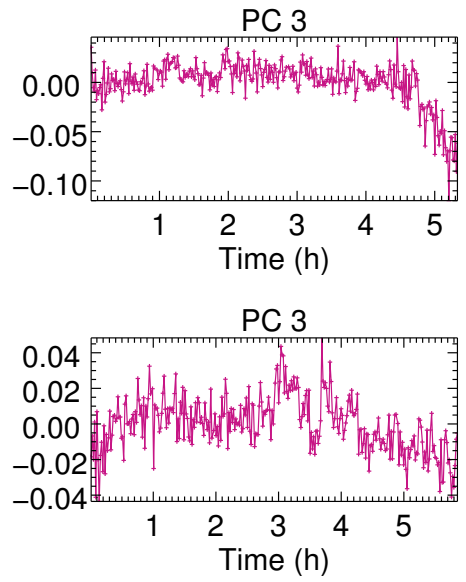

Figure 12. Most important principal components retained for for 2013 December 22 (top) and 2014 October 10 (bottom).

McQuillan, A., Mazeh, T., \& Aigrain, S. 2014, ApJS, 211, 24

Metchev, S., Apai, D., Radigan, J., et al. 2013, AN, 334, 40

Metchev, S. A., Heinze, A., Apai, D., et al. 2015, ApJ, 799, 154

Morley, C. V., Marley, M. S., Fortney, J. J., \& Lupu, R. 2014, ApJL, 789, L14

Naud, M.-E., Artigau, É., Malo, L., et al. 2014, ApJ, 787, 5 Naud, M.-E., Artigau, É., Doyon, R., et al. 2017, AJ, 154, 3

Press, W. H., Teukolsky, S. A., Vetterling, W. T., \& Flannery, B. P. 1992, Cambridge: University Press, —c1992, 2nd ed.

Puget, P., Stadler, E., Doyon, R., et al. 2004, Proc. SPIE, 5494, 978

Radigan, J. 2014, ApJ, 797, 120

Radigan, J., Jayawardhana, R., Lafrenière, D., et al. 2012, ApJ, 750,105
Radigan, J., Lafrenière, D., Jayawardhana, R., \& Artigau, É. 2014, ApJ, 793, 75

Robinson, T. D., \& Marley, M. S. 2014, ApJ, 785, 158

Rowe, J. F., Bryson, S. T., Marcy, G. W., et al. 2014, ApJ, 784, 45

Showman, A. P., \& Kaspi, Y. 2013, ApJ, 776, 85

Simon, A. A., Rowe, J. F., Gaulme, P., et al. 2016, ApJ, 817, 162

Snellen, I. A. G., Brandl, B. R., de Kok, R. J., et al. 2014,

Nature, 509, 63

Wilson, P. A., Rajan, A., \& Patience, J. 2014, A\&A, 566, A111

Yang, H., Apai, D., Marley, M. S., et al. 2016, ApJ, 826, 8

Zhou, Y., Apai, D., Schneider, G. H., Marley, M. S., \& Showman, A. P. 2016, ApJ, 818, 176

Zhu, C., Byrd, R. H., Lu, P., \& Nocedal, J. 1997, ACM Transactions on Mathematical Software (TOMS), 23, 550 